\title{
Temperature dependence of the positronium work function in aluminum
}

\author{
B. K. Panda, C. D. Beling, and S. Fung \\ Department of Physics, University of Hong Kong, Hong Kong
}

(Received 17 February 1994)

\begin{abstract}
The electron and positron chemical potentials and their volume derivatives are calculated in $\mathrm{Al}$ using the first-principles pseudopotential technique and the frozen-core approximation method, respectively. The positron deformation potential of $-8.03 \mathrm{eV}$ obtained by this method agrees very well with that found in other first-principles calculations. The variation of the positronium work function with temperature is calculated by including the thermal lattice vibrations into the electron and positron potentials. It is observed that the temperature dependence of the positronium work function does not arise entirely from the volume expansion of the lattice as has been suggested in the past. Proper inclusion of the nonzero-temperature derivative of the positronium work function at constant volume leads to good agreement between the positron deformation potential as obtained from positronium work-function data and present theoretical values.
\end{abstract}

\section{INTRODUCTION}

That an electron cannot escape from a solid without additional energy was first understood in terms of its work function in the solid by Einstein in $1905 .^{1} \mathrm{~A}$ complete many-body theory of the electron work function in a solid, however, had to await the work of Lang and Kohn in $1971 .^{2}$ In their theory the work function $\left(\phi_{-}\right)$is separated into the bulk electron chemical potential $\left(\mu_{-}\right)$, which is the difference of the ground state energies before and after the removal of an electron from the solid, and the surface dipole potential $(\Delta)$ which arises from the electrons "spilling out" beyond the surface planes. The electron is electrically repelled back into the solid by the surface dipole potential. The work function is thus expressed as $\phi_{-}=-\mu_{-}+\Delta$. On the other hand, low energy positrons from a positron beam after implantation into the near surface region of single crystal metals thermalize in a short time, and in most cases a fraction of them escape spontaneously into the vacuum. ${ }^{3}$ This phenomenon arises because the positron is attracted by the same surface dipole potential $\Delta$ into the vacuum. The positron work function $\left(\phi_{+}\right)$may also be expressed in terms of the positron chemical potential $\left(\mu_{+}\right)$and the surface dipole potential with an opposite sign because of the attraction, i.e., $\phi_{+}=-\mu_{+}-\Delta$. In such measurements it is also seen for most metals that some of the thermalized positrons pick up electrons to form positronium atoms which escape spontaneously into the vacuum. ${ }^{3}$ Since the positronium atom is electrically neutral, the emission is not controlled by the surface dipole potential. The positronium work function given by $\phi_{\mathrm{ps}}=\phi_{-}+\phi_{+}-6.8 \mathrm{eV}$ is thus a bulk property, the surface dipole potential canceling to give $\phi_{\mathrm{ps}}=-\mu_{-}-\mu_{+}-6.8 \mathrm{eV}$.

Measurements of the positronium work function in aluminum at different temperatures have been performed. ${ }^{4,5}$ It is observed that the positronium work function has a linear temperature dependence. The temperature influences $\phi_{\mathrm{ps}}$ through (i) thermal expansion of the volume $\boldsymbol{\Omega}$ of the solid and (ii) thermal vibrations of the atoms around their mean positions. The temperature coefficient for the positronium work function at constant pressure is given by

With the deformation potential characterizing the positron-phonon coupling and the thermal expansion coefficient defined as $E_{d}=-\Omega\left(\frac{\partial \phi_{\mathrm{ps}}}{\partial \Omega}\right)_{T}$ and $\beta=\frac{1}{\Omega}\left(\frac{\partial \Omega}{\partial T}\right)_{P}$, respectively, Eq. (1.1) is rewritten as

$$
\frac{d \phi_{\mathrm{ps}}}{d T}=-\beta E_{d}+\left(\frac{\partial \phi_{\mathrm{ps}}}{\partial T}\right)_{\Omega} .
$$

The positron deformation potential can thus be obtained from this equation provided the value of the second term is known. Unfortunately, there is no experimental method available to measure its contribution. However, Gullikson and Mills ${ }^{5}$ in their measurement argued that the contribution of the second term is exceedingly small and the temperature dependence of the positronium chemical potential is entirely due to the volume expansion of the solid. With this assumption and with the known value of $\beta$ for $\mathrm{Al}$ at room temperature, $E_{d}$ has been calculated from the slope of $\frac{d \phi_{\mathrm{ps}}}{d T}$ to have the value $-11.5 \mathrm{eV}$, whereas the other experimental data by Rosenberg, Howell, and Fluss ${ }^{4}$ give $-13.7 \mathrm{eV}$. The value calculated in the linear muffin-tin-orbital method within the atomic-spheres approximation (LMTO-ASA) by Boev, Puska, and Nieminen ${ }^{6}$ is $-7.7 \mathrm{eV}$ and by Puska et al. ${ }^{7}$ is $-7.85 \mathrm{eV}$. The simple uniform electron gas model by Bergersen et $a .^{8}$ gives $-8.6 \mathrm{eV}$, the magnitude of which is also quite small compared to the experimental value. The disagreement of the experimental data with the theoretical data could arise from two sources. First, a large scatter in the data may have resulted in a wrong slope being assigned, thus giving too small a value for $E_{d}$. The second and more likely explanation is that the neglect of the temperature derivative of the positronium work function at a constant volume (if it has the same temperature dependence as that of $\beta$ ) may result in an incorrectly calculated deformation potential. Although Boev et $a .^{6}$ have given expressions for $\left(\frac{\partial \mu_{-}}{\partial T}\right)_{\Omega}$ and $\left(\frac{\partial \mu_{+}}{\partial T}\right)_{\Omega}$, they gave no quantitative values. On the basis of physical arguments they have speculated that since $\left(\frac{\partial \mu_{-}}{\partial T}\right)_{\Omega}>0$ 
and $\left(\frac{\partial \mu_{+}}{\partial T}\right)_{\Omega}<0$ there is some cancellation in $\left(\frac{\partial \phi_{\mathrm{ps}}}{\partial T}\right)_{\Omega}$ resulting in a small value. However, it cannot be assumed that $\left(\frac{\partial \phi_{\mathrm{ps}}}{\partial T}\right)_{\Omega}$ would vanish identically. In the present work we have computed the contributions of the lattice vibrations to $\phi_{\mathrm{ps}}$ using a different method. We shall show that the temperature coefficient (at constant volume) of the positron chemical potential is lower than that of the electron chemical potential, suggesting that the deformation potential derived from the experimental data, on the assumption that $\left(\frac{\partial \phi_{\mathrm{ps}}}{\partial T}\right)_{\Omega}$ can be neglected, is higher than the actual value.

\section{THEORY}

The evaluation of the electron and positron chemical potentials is based on the local density approximation (LDA). ${ }^{6,7}$ The electron band energies are solved from the $a b$ initio pseudopotential theory. The self-consistent pseudopotential with electron charge density $\rho(\mathbf{r})$ is given by

$$
\begin{aligned}
V_{\mathrm{ps}}(\mathbf{r}) & =V_{\text {ion }}(\mathbf{r})+V_{H}(\rho(\mathbf{r}))+V_{\mathbf{x c}}(\rho(\mathbf{r})) \\
& =V_{\text {Coulomb }}^{-}(\mathbf{r})+V_{\mathbf{x c}}(\rho(\mathbf{r})),
\end{aligned}
$$

where $V_{\text {ion }}$ is the combination of local $V_{\text {ion }}^{L}$ and nonlocal $V_{\text {ion }}^{\mathrm{NL}}$ pseudopotentials, ${ }^{9} V_{H}$ is the Hartree potential, and $V_{\text {xc }}$ the exchange-correlation potential. ${ }^{10}$ Since $V_{\text {ion }}$ and $V_{H}$ are both Coulombic in nature, we have denoted their sum by $V_{\text {Coulomb. The electron chemical potential is }}^{-}$ defined as the sum of the Fermi energy and the exchangecorrelation potential calculated with the charge density $\left(\rho_{0}\right)$ at the surface of the Wigner-Seitz cell ${ }^{7}$

$$
\mu_{-}=E_{F}+V_{\mathrm{xc}}\left(\rho_{0}\right)-\left\langle V_{\text {Coulomb }}^{-}\right\rangle .
$$

Here $\left\langle V_{\text {Coulomb }}^{-}\right\rangle$is the crystal averaged Coulomb potential evaluated on the surface of the Wigner-Seitz cell. Since the present calculation is based on the pseudopotential method, there is an energy shift between extended cores and the pointlike ones. ${ }^{11}$ The electron chemical potential is corrected for this shift, which is given by

$$
\Delta E=\frac{1}{\Omega} \int\left(V_{\text {ion }}^{L}(\mathbf{r})+\frac{Z e^{2}}{r}\right) d^{3} r,
$$

where $\Omega$ is the unit cell volume. ${ }^{11}$ This term has been evaluated in the work function calculation of $\mathrm{Si}$ by Pennetta $^{12}$ by using core electronic charge density, which may not be correct for all pseudopotential calculations because of the nonuniqueness of the pseudopotential.

The positron potential is constructed in the model where there is one positron in the many-electron system. In the frozen-core approximation the positron potential is purely Coulombic in nature, with repulsion to the point nuclei and attraction to the valence and frozen-core electron charge densities. The valence and core charge densities are calculated from the pseudopotential method and the Roothaan-Hartree-Fock wave functions, ${ }^{13}$ respectively. In addition to this there is an attractive shortrange electron-positron correlation potential $V_{\text {corr }}$ :

$$
V_{+}(\mathbf{r})=V_{\text {Coulomb }}^{+}(\mathbf{r})+V_{\text {corr }}(\rho(\mathbf{r})) .
$$

The correlation potential is calculated in a homogenous electron gas based on the LDA. ${ }^{14}$ The positron chemical potential is defined as

$$
\mu_{+}=E_{0}+E_{\text {corr }},
$$

where $E_{0}$ is the zero point energy relative to the average electrostatic potential $\left\langle V_{\text {Coulomb }}^{+}\right\rangle$on the surface of the Wigner-Seitz cell. With the positron ground state wave function at zero temperature $\left[\Psi_{+}(\mathbf{r})\right], E_{0}$ is defined as ${ }^{6,7}$

$$
\begin{aligned}
E_{0}= & \int d^{3} r \Psi_{+}^{*}(\mathbf{r})\left[-\frac{1}{2} \nabla^{2}+V_{\text {Coulomb }}^{+}(\mathbf{r})\right] \Psi_{+}(\mathbf{r}) \\
& -\left\langle V_{\text {Coulomb }}^{+}\right\rangle .
\end{aligned}
$$

The electron-positron correlation contribution to the positron chemical potential is

$$
E_{\text {corr }}=\int d^{3} r\left|\Psi_{+}(\mathbf{r})\right|^{2} V_{\text {corr }}(\rho(\mathbf{r})) .
$$

There are two major corrections to the change of the energy levels in the solid resulting from the thermal vibration of the atoms. Fan ${ }^{15}$ proposed a temperaturedependent self-energy correction involving an electronphonon coupling term in the second order to give a shift in the energy levels. In the other method the lattice potentials are corrected by the temperature-dependent Debye-Waller factor $[W(G, T)],{ }^{16}$ which is in turn related to the total mean squared displacement of the atoms. ${ }^{17}$ Allen and Heine ${ }^{18}$ combined both the self-energy and Debye-Waller corrections into one expression involving the second order in atomic displacement. The expressions given by Boev et al. ${ }^{6}$ are based on this scheme. We have adopted the second procedure in the present work. The self-consistent electron pseudopotential of Eq. (2.1) is Fourier transformed to the reciprocal space to obtain $V_{\text {ps }}(\mathbf{G})$ and then multiplied by $\exp [-2 W(G, T)]$ to include lattice vibrations,

$$
V(\mathbf{G}, T)=V_{\mathrm{ps}}(\mathbf{G}) \exp [-2 W(G, T)] .
$$

The Schrödinger equation is solved in the momentum space to get both the band energy $E_{n \mathbf{k}}$ and wave function $\Psi_{n \mathbf{k}}(\mathbf{r})$ with $n$ and $\mathbf{k}$ being the band index and the wave vector, respectively. The charge density is calculated at different temperatures as

$$
\rho(\mathbf{r})=\sum_{n \mathbf{k}} F_{-}\left(E_{n \mathbf{k}}, T\right)\left|\Psi_{n \mathbf{k}}(\mathbf{r})\right|^{2} .
$$

$F_{\text {- }}$ is the Fermi-Dirac distribution function. The electron chemical potential at a given temperature is obtained from the recalculated Fermi energy, the Coulomb potential, and the correlation potential in a same manner described for the zero-temperature formalism.

The positron potential at a finite temperature is also obtained in a similar manner to the electron case. The positron potential $V_{+}(\mathbf{r})$ in Eq. (2.4) is first Fourier transformed to the reciprocal space and then corrected for the Debye-Waller factor. The wave vector of the positron at finite temperature is given by $k_{+}=\left(\frac{3 k_{B} T m^{*}}{\hbar^{2}}\right)^{1 / 2}$ where $m^{*}$ is the effective mass of the positron. In addition to this it is taken that the positron behaves like a classical particle with Boltzmann distribution. With these extra conditions the zero-point energy of the positron in Eq. (2.6) is modified as 


$$
\begin{aligned}
E_{0}= & \sum_{\mathbf{k}_{+}} F_{+}\left(\mathbf{k}_{+}, T\right) \int d^{3} r \Psi_{\mathbf{k}_{+}}^{*}(\mathbf{r}) \\
& \times\left[-\frac{1}{2} \nabla^{2}+V_{\text {Coulomb }}^{+}(\mathbf{r})\right] \Psi_{\mathbf{k}_{+}}(\mathbf{r})-\left\langle V_{\text {Coulomb }}^{+}\right\rangle,
\end{aligned}
$$

where $F_{+}\left(\mathbf{k}_{+}, T\right)$ is the Maxwell-Boltzmann distribution function. Like $E_{0}$, the correlation energy in Eq. (2.7) is modified as

$$
E_{\text {corr }}=\sum_{\mathbf{k}_{+}} F_{+}\left(\mathbf{k}_{+}, T\right) \int d^{3} r \Psi_{\mathbf{k}_{+}}^{*}(\mathbf{r}) V_{\text {corr }}(\rho(\mathbf{r})) \Psi_{\mathbf{k}_{+}}(\mathbf{r})
$$

\section{RESULTS AND DISCUSSIONS}

The pseudopotential calculation is carried out at the zero-temperature lattice constant 7.656 a.u. to obtain the electron chemical potential. The self-consistent pseudocharge-densities together with the core charge densities are used in the frozen-core positron potential to obtain the positron chemical potential. Both the electron and positron chemical potentials are presented in Table I. We also present the electron and positron chemical potentials obtained by Boev et al. ${ }^{6}$ and Puska et al. ${ }^{7}$ in their LMTO-ASA calculation in Table I. The chemical potentials obtained in this calculation are in good agreement with their values. The calculation is carried out at slightly different lattice constants around the zerotemperature lattice constant to obtain the volume derivative of the electron and positron chemical potentials. The different contributions to the positron deformation potential, $E_{d}$, are given in Table I. We also present the corresponding contributions obtained previously by both Boev et al. in their LMTO-ASA calculation and the superposition of free atoms method. The calculated values of Bergersen et al. ${ }^{8}$ using a uniform electron gas model are also tabulated in Table I. In our calculation we have obtained the volume coefficients of the Fermi energy, the exchange-correlation potential, and the average Coulomb potential energy as $-13.23 \mathrm{eV}, 3.57 \mathrm{eV}$, and $2.52 \mathrm{eV}$, respectively. Unfortunately the corresponding data in other calculations are not available for comparison. It is interesting to see that the lattice expansion coefficient of the electron chemical potential lies between the values calculated in the LMTO-ASA method and in the uniform electron gas model. We have used soft-core pseudopotentials which are stronger than the potentials used in the uniform electron gas model, but weaker than those used in LMTO-ASA method. In general, with more interstitial space available, the electron charge density decreases with volume expansion. The exchange-correlation potential in turn becomes less attractive resulting in the observed positive volume coefficient of $3.25 \mathrm{eV}$. Since in the pseudopotential method the charge density is primarily distributed in the interstitial region, the electron density decreases slowly compared to the LMTO-ASA calculation. For this reason the volume derivative of the correlation potential in the LMTO-ASA calculation is larger than our result. The volume expansion of the lattice also decreases the Fermi energy of the solid, giving a negative volume derivative, and it is this rapid decrease in Fermi energy which is mainly responsible for the mag- nitude and sign of the volume derivative of the electron chemical potential. In the uniform electron gas distribution picture, the decrease of the charge density at the Wigner-Seitz radius is slower than in the pseudopotential scheme. This results in a smaller value of the exchangecorrelation potential component and a larger value of the volume derivative of the electron chemical potential.

The volume derivative of the positron chemical potential in the present calculation lies between the values calculated by LMTO-ASA and uniform electron density methods, due to the same reason as given for the electron chemical potential. Like the electron exchangecorrelation potential, the positron correlation potential is attractive in nature. Hence the decrease in the charge density at the interstitial region results in a positive contribution to the volume coefficient of the positron correlation energy. But the lattice expansion decreases the positron potential, which results in lowering the zeropoint energy. In the present calculation the cancellation of the nuclear and frozen-core charge densities is more rapid, to make the positron potential weaker than in the LMTO-ASA calculation, but strong enough compared to the uniform electron gas result.

The temperature derivative of the electron chemical potential is calculated first by obtaining the selfconsistent pseudopotentials at zero-temperature lattice constant and then by modifying them by the thermal vibrations of the atoms at different temperatures. We have varied the temperature from 100 to $600 \mathrm{~K}$ in this calculation. It is observed that $\mu_{-}$varies linearly with temperature. The temperature coefficients of $E_{F}$ and $V_{\mathbf{x c}}\left(\rho_{0}\right)$ are found to be $0.241 \mathrm{meV} \mathrm{K}^{-1}$ and $0.014 \mathrm{meV} \mathrm{K}^{-1}$, respectively. There is no appreciable change of the average potential. The value of $\left(\frac{\partial \mu_{-}}{\partial T}\right)_{\Omega}$ is calculated as 0.255 $\mathrm{meV} \mathrm{K}^{-1}$. Our positive temperature coefficient of the electron chemical potential agrees with the prediction of Boev et al. ${ }^{6}$ With increasing temperature the potential is damped by the vibrations of the atoms, to result in lowering the charge density in the interstitial region. From our calculation we find that the thermal lattice vibrations of the atoms in the solid do not change the valence and core charge densities appreciably, but increase the Fermi energy.

The positron chemical potentials are computed for different temperatures with the positron wave vector $\left(k_{+}\right)$ calculated by taking the effective mass of the positron to be $1.15 .^{19}$ They are found to decrease linearly with the temperature. The values of $\frac{\partial E_{0}}{\partial T}$ and $\frac{\partial E_{\mathrm{corr}}}{\partial T}$ are found to be $-0.3962 \mathrm{meV} \mathrm{K}^{-1}$ and $0.0011 \mathrm{meV} \mathrm{K}^{-1}$, respectively. The change in average chemical potential is found to be negligibly small. The value of $\frac{\partial \mu_{+}}{\partial T}$ is calculated to be $-0.3951 \mathrm{meV} \mathrm{K}^{-1}$. Since the potentials are damped by the lattice vibrations, the positron chemical potential is expected to increase with increasing temperature as in the case of the electron chemical potentials. Although the shift in mean atomic positions affects the positron distribution in every cell, the lattice vibrations can squeeze the positron from a cell that is contracted into an expanded neighboring cell. This may cancel the damping produced by the Debye-Waller factor and the positrons can lower 
TABLE I. The contributions of the electron and positron chemical potentials to the positron deformation potential $E_{d}$. SFA is the superposition of free atoms and UEGM the uniform electron gas model. The values of $\mu_{-}$and $\mu_{+}$are for the zero-temperature lattice constant.

\begin{tabular}{cccccccc}
\hline \hline & $\begin{array}{c}\mu_{-} \\
(\mathrm{eV})\end{array}$ & $\begin{array}{c}\mu_{+} \\
(\mathrm{eV})\end{array}$ & $\begin{array}{c}\Omega \frac{\partial \mu_{-}}{\partial \Omega} \\
(\mathrm{eV})\end{array}$ & $\begin{array}{c}\Omega \frac{\partial E_{0}}{\partial \Omega} \\
(\mathrm{eV})\end{array}$ & $\begin{array}{c}\Omega \frac{\partial E_{\text {corr }}}{\partial \Omega} \\
(\mathrm{eV})\end{array}$ & $\begin{array}{c}\Omega \frac{\partial \mu_{+}}{\partial \Omega} \\
(\mathrm{eV})\end{array}$ & $\begin{array}{c}E_{d} \\
(\mathrm{eV})\end{array}$ \\
\hline LMTO-ASA $^{\mathrm{a}}$ & -0.74 & -3.35 & -6.00 & -3.42 & 1.72 & -1.70 & -7.7 \\
LMTO-ASA $^{\mathrm{b}}$ & -0.76 & -3.65 & -6.02 & & & -1.83 & -7.85 \\
$\quad \begin{array}{l}\text { SFA } \\
\text { (a) }\end{array}$ & & & & -2.76 & 1.74 & & -8.03 \\
$\begin{array}{c}\text { Present method } \\
\text { UEGM }\end{array}$ & -0.58 & -3.35 & -7.14 & -2.43 & 1.54 & -0.89 & -8.60 \\
\hline \hline
\end{tabular}

\footnotetext{
${ }^{\mathrm{a}}$ Reference 6 .

${ }^{\mathrm{b}}$ Reference 7 .

${ }^{\mathrm{c}}$ Reference 8 .
}

their energies to adjust to the vibrations of the atoms.

Taking the calculated values of the temperature derivatives of the electron and positron chemical potentials, the value of $\left(\frac{\partial \phi_{\mathrm{ps}}}{\partial T}\right)_{\Omega}$ is found to be $0.1401 \mathrm{meV} \mathrm{K}^{-1}$. With the lattice thermal expansion $\beta$ at $300 \mathrm{~K}$ taken as $70.8 \times 10^{-6} \mathrm{~K}^{-1},{ }^{20}$ the value of $\frac{d \phi_{\mathrm{ps}}}{d T}$ is $0.71 \mathrm{meV} \mathrm{K}^{-1}$ which is slightly less than the experimental data of Gullikson and Mills, ${ }^{5}$ but significantly lower than the data of Rosenberg et al. ${ }^{4}$ The recalculated deformation potential from the data of Gullikson and Mills is $-8.61 \mathrm{eV}$, which is the same as that calculated in the uniform electron gas model and is also in reasonable agreement with the value of $-8.03 \mathrm{eV}$ found in this work. At this stage we do not justify that the uniform electron gas model is better than the present or the LMTO-ASA calculations for the positronium work function. We simply point out that inclusion of the term $\left(\frac{\partial \phi_{\mathrm{ps}}}{\partial T}\right)_{\Omega}$ leads to a removal of the previous discrepancies between theoretically and experimentally obtained positron deformation potentials.
Uncertainties that exist in the experimental values of $E_{d}$ (Refs. 5 and 4) and the neglect in the present work of the self-energy correction contribution are factors which have to be addressed before more concrete conclusions can be drawn. As a point of interest, it is noted that the theoretical $E_{d}$ values compiled by Boev et al. ${ }^{6}$ for heavier elements come into better agreement with experiment without any lattice vibration corrections. For example, $\mathrm{Pb}$ with a theoretical $E_{d}$ of $-6.39 \mathrm{eV}$ is compared with the experimental values of $-6.11 \mathrm{eV},{ }^{21}$ and $-6.13 \mathrm{eV}{ }^{4}$ While we would suggest calculations be made of the lattice vibration effect for these elements, we point out that the Debye-Waller correction will be smaller for heavier atoms. In aluminum, which is a light element, the effect of lattice vibrations is seen more clearly.

\section{ACKNOWLEDGMENTS}

Dr. M. Šob is thanked for helpful comments on this work. One of us, B.K.P, would like to thank the University of Hong Kong for financial support.
${ }^{1}$ A. Einstein, Ann. Phys. (Leipzig) 17, 132 (1905).

${ }^{2}$ N. D. Lang and W. Kohn, Phys. Rev. B 3, 1215 (1971).

${ }^{3}$ A. P. Mills, Jr. in Positron Solid-State Physics, edited by W. Brandt and A. Dupasquier (North-Holland, Amsterdam, 1983), p. 432.

${ }^{4}$ I. J. Rosenberg, R. H. Howell, and M. J. Fluss, Phys. Rev. B 35, 2083 (1987).

${ }^{5}$ E. M. Gullikson and A. P. Mills, Jr., Phys. Rev. B 35, 8759 (1987).

${ }^{6}$ O. V. Boev, M. J. Puska, and R. M. Nieminen, Phys. Rev. B 36, 7786 (1987).

${ }^{7}$ M. J. Puska, P. Lanki, and R. M. Nieminen, J. Phys. Condens. Matter 1, 6081 (1989).

${ }^{8}$ B. Bergersen, E. Pajanne, P. Kubica, M. J. Scott, and C. H. Hodges, Solid State Commun. 15, 1377 (1974).

${ }^{9}$ G. B. Bachelet, D. R. Hamann, and M. Schlüter, Phys. Rev. B 26, 4199 (1982).

${ }^{10}$ D. M. Ceperley and B. J. Alder, Phys. Rev. Lett. 45, 566 (1980); parametrized in J. Perdew and A. Zunger, Phys. Rev. B 23, 5048 (1981).

11 J. Ihm, A. Zunger, and M. L. Cohen, J. Phys. C 12, 4409 (1979).

12 C. Pennetta, Solid State Commun. 77, 159 (1991).
${ }^{13}$ E. Clementi and C. Roetti, At. Data Nucl. Data Tables 14, 177 (1974).

14 J. Arponen and E. Pajanne, Ann. Phys. (N.Y.) 121, 343 (1979); J. Phys. F 9, 2359 (1979); parametrized in P. A. Sterne, J. H. Kaiser, J. C. O'Brien, and R. H. Howell, in Positron Annihilation, Vol. 105-110 of Materials Science Forum, edited by Zs. Kajcsos and Cs. Szeles (Trans. Tech, Aedermannsdorf, 1992), p. 469.

${ }^{15}$ H. Y. Fan, Phys. Rev. 82, 900 (1951).

${ }^{16}$ M. L. Cohen and J. R. Chelikowsky, in Electronic Structure and Optical Properties of Semiconductors, edited by M. Cardona (Springer-Verlag, Berlin, 1988), p. 182; E. Antoncik, Czech. J. Phys. 5, 449 (1955); C. Keffer, T. M. Hayes, and A. Bienenstock, Phys. Rev. Lett. 21, 1676 (1968).

${ }^{17}$ L. C. Feldman and J. W. Mayer, Fundamentals of Surface and Thin Films Analysis (North-Holland, Amsterdam $1986)$, p. 158.

${ }^{18}$ P. B. Allen and V. Heine, J. Phys. C 9, 2305 (1976).

${ }^{19}$ P. Kubica and M. J. Scott, J. Phys. F 4, 1969 (1974).

${ }^{20}$ N. W. Ashcroft and N. D. Mermin, Solid State Physics (Holt, Rinehart and Winston, New York, 1976), p. 496.

${ }^{21}$ P. J. Schultz and K. G. Lynn, Phys. Rev. B 26, 2390 (1982). 